RAP CONFERENCE PROCEEDINGS, VOL. 5, PP. 38-43, 2020

ISSN 2737-9973 (ONLINE)| DOI: 10.37392/RAPPROC.2020.10

RAP-PROCEEDINGS.ORG

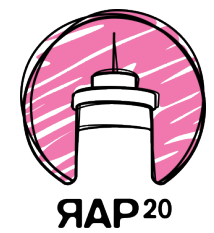

\title{
FEATURES OF DNA REPAIR IN DERMAL FIBROBLASTS IN PATIENTS WITH BREAST CANCER AND PERSONS WITH MEDICAL HISTORY OF CANCER
}

\author{
Aleksandra Nozdracheva ${ }^{1,2^{*}}$, Nadezhda Pleskach', Mirya Kuranova ${ }^{1}$ \\ ${ }^{1}$ Institute of Cytology of the Russian Academy of Science, Saint-Petersburg, Russia \\ ${ }^{2}$ Peter the Great St. Petersburg Polytechnic University, Saint-Petersburg, Russia
}

\begin{abstract}
In the cell lines of dermal fibroblasts of 30 and 55 year-old patients with breast cancer (BC) and in three patients with medical history of cancer, the features of DNA repair were studied. The amount, intensity of fluorescence and area of foci of protein $53 \mathrm{BP} 1$ and histone $\gamma \mathrm{H}_{2} \mathrm{AX}$ were investigated at different time parameters of the field of irradiation of cells at a dose of $2 \mathrm{~Gy}$. A line from a 30-year-old patient with no history of cancer was used as a healthy donor. The results showed that the reparative curve of a healthy donor differed from the reparative curves of the remaining lines. The cells of a young patient with breast cancer had more serious violations of the DNA repair processes. Also, the results of the work showed that histone $\gamma \mathrm{H} 2 \mathrm{AX}$ is more specific for breast cancer.
\end{abstract}

Keywords: Breast cancer, DNA repair, $53 B P 1, \gamma H 2 A X$

\section{INTRODUCTION}

Maintaining the stability and integrity of the genome is provided by several key signaling pathways working together. Defective components in the mechanisms of damage and DNA repair are the main cause of the development and progression of various types of cancer, and breast cancer is no exception.

Continuous exposure to internal and external stressors, such as reactive oxygen species (ROS) and ionizing radiation, causes DNA damage. Damage to DNA can be either recoverable or non-recoverable. The most common DNA damage occurrences are base mismatch or adducts, insertion/deletion, O6 alkylguanine formation, DNA cross-linking, singlestrand breaks (SSB) and double-strand breaks (DSBs). In response to DNA damage, cells activate the control points of the cell cycle and the DNA repair mechanism is launched to completely eliminate the damage. If the damage is too severe, the apoptosis program starts.

The cell response to DNA damage is a complex signaling network and is commonly called the DNA damage response (DDR). DDR is necessary to maintain genome stability and integrity and to continue replication and transcription [1].

To restore one DSB, it is necessary to hydrolyze more than 104 ATP molecules; therefore, timely and correct restoration of various types of DNA damage is extremely important, given the energy costs of cell cleaning [2].

Genome maintenance and cancer prevention is driven by genes encoding important components of DDR and, in particular, DNA repair pathways. These genes are one of the most frequently mutated genes in cancer $[3,4]$.

Mutations in the BRCA gene account for about 20\% of all inherited forms of breast cancer. Thanks to full genomic sequencing, other breast cancer susceptibility genes (CHEK2, PALB2, ATM and TP53) have been identified, which together can contribute up to $30 \%$ of all inherited forms of breast cancer [5].

One of the important DNA repair and genome stability genes is the ATM gene (OMIM \#607585), which encodes a key repair kinase for DSBs. In colorectal cancer, which is the most common of all cancers, the ATM gene is a target for mutation, as well as a modifier gene with a high risk of the disease development due to its polymorphism in the ATM variant $\mathrm{D} 1853 \mathrm{~N}$ [6]. In the Spanish population, about $2 \%$ of breast cancer cases are caused by mutations in the ATM, HBOC, and BRCAX genes [7].

$53 \mathrm{BP} 1$ is a chromatin-binding protein that regulates the repair of double-stranded DNA breaks by suppressing nucleolytic DNA end resection. The manner in which the proteins of the $53 \mathrm{BP} 1$ pathway protect the ends of DNA is currently unknown, but there is evidence in favor of a model in which the 53BP1 effector complex, shieldin, protects the ends of DNA to provide DNA repair [8].

Despite significant advances in the diagnosis, prognosis and treatment of breast cancer, tumors have limited therapeutic options. Many treatment strategies attempt to investigate the limitations of the DNA damage response (DDR) in tumor cells in order to selectively eliminate them. Tumors with worse prognosis (grade 3 tumor and triple negative) showed reduced expression of tBRCT genes, in particular,

* surupchi@mail.ru 
PAXIP1 and TP53BP1. The state of tumor's both genes can affect the prognosis. The levels of the PAXIP1 and $53 \mathrm{BP} 1$ protein follow the results of gene expression, i.e., correlate in nature, and also decrease in more advanced tumors [9].

Histone $\mathrm{H} 2 \mathrm{AX}$ is a target protein for ATM kinase and is phosphorylated in response to double-stranded DNA breaks. The latter, in turn, are part of the oncogenic transformation. The detection of $\gamma \mathrm{H} 2 \mathrm{AX}$ has the potential to serve as a biomarker for the conversion of normal tissue to precancerous and malignant tissue. The larger the tumor, the degree and number of affected lymph nodes, the more the $\gamma \mathrm{H} 2 \mathrm{AX}$ expression. High estrogen and progesterone receptor values are largely associated with lower $\gamma \mathrm{H} 2 \mathrm{AX}$ values.

DNA repair can be assessed by constructing repair curves after exposure of cells to ionizing radiation at different time intervals [10, 11].

\section{MATERIALS AND METHODS}

\subsection{Study group selection}

We studied three groups:

1. Patients with breast cancer: two patients of 30 (BC30SP1) and 55 (BC55SP1) years of age.

2. Breast cancer risk group: a 30 year-old person with 5382insC mutation in the BRCA1 gene (BRCA1SP) and two mothers of patients with ataxia-telangiectasia - 47 (AT8MSP) and 45 (AT9MSP) years old. Heterozygous carriers of mutations in the ATM gene are at risk of tumors, including breast cancer.

3. Healthy donor without breast cancer or a history of oncological diseases who was 30 years old (N9SP).

The group of patients conducting the research: "patients with breast cancer", "person with history of cancer" and "healthy donors". The patients group included two patients with breast cancer 30 and 55 years old (BC30SP1 and BC55SP1). The "person with history of cancer» consisted of three persons. This is a 30 year old woman with a 5382incC mutation in the BRCA1 gene (BRCA1SP) with history of breast cancer. This woman's sister and mother were diagnosed with breast cancer. And two mothers of patients with ataxia telangiectasia 47 and 45 years old (AT8MSP and AT9MSP). These two mothers also have a history of cancer (stomach cancer, breast cancer, bowel cancer).This according to the data, heterozygous carriers during mutations in the ATM gene are at risk of a tumor.

For two patients (AT8SP and AT9SP) molecular genetic analysis was done (whole genome sequencing and NGS sequencing), but no pathogenic substitutions in the ATM gene were detected.

The study of the DNA of the family of the AT8SP patient using indirect polymorphic markers D11S384, D11S2179 and D11S1294 linked to the ATM gene showed that the patient AT8SP is a carrier of a mutation in the ATM gene.

Also for two patients (AT8SP and AT9SP) cell analysis to identify the active form of ATM kinase (pATMSer1981) was done. Cell of AT8SP patient, the active form of ATM kinase was not detected, cell of AT9SP patient, the proportion of cells that were unable to form and delayed the child's response to DNA damage was revealed [12].

The healthy donor was the 30 year-old donor who did not have breast cancer and did not have a history of oncological diseases (N9SP).

All patients and a healthy donor given inform consent.

\subsection{Obtainment cell lines}

Cell lines of dermal fibroblasts BRCA1SP, BC30SP and $\mathrm{BC}_{55} \mathrm{SP}$ were obtained by the migration method from the post-operative material. Lines of dermal fibroblasts N9SP, AT8MSP and AT9MSP were obtained from a skin biopsy, which was performed under local anesthesia. Skin biopsies were crushed into small pieces and placed in Petri dishes, filled with complete MEM growth medium (Gibco, USA) containing $15 \%$ bovine fetal serum (Gibco, USA), 100 $\mathrm{IU} / \mathrm{ml}$ penicillin, $100 \mu \mathrm{g} / \mathrm{ml}$ streptomycin (Gibco, USA), $25 \mu \mathrm{g} / \mathrm{ml}$ fungicon, $0.3 \mathrm{mg} / \mathrm{ml} \mathrm{L-glutamine,} \mathrm{and}$ cultured in a $\mathrm{CO} 2$ incubator at $+37^{\circ} \mathrm{C}$ in an atmosphere with 5\% $\mathrm{CO} 2$ until a subconfluent monolayer was obtained. Next, the cells were rinsed with a solution of phosphate-buffered saline (PBS), removed from Petri dishes using $0.25 \%$ trypsin-versene solution (Gibco, USA) and scattered on coverslips (Carl Zeiss, Germany).

\subsection{DNA damage}

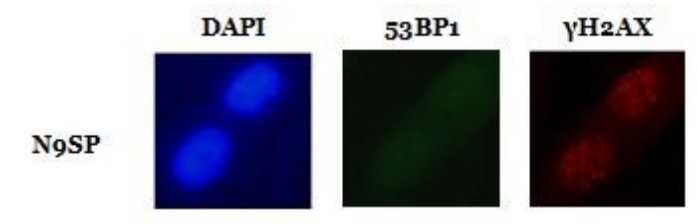

BRCA1SP
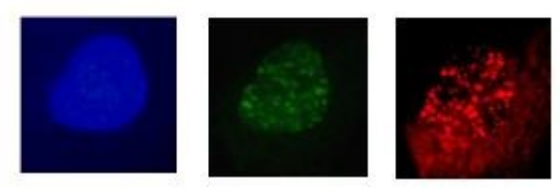

AT8MSP
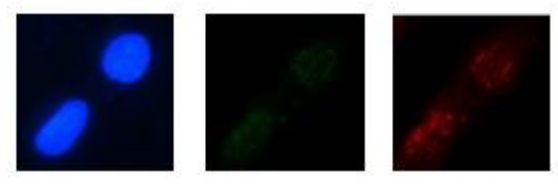

AT9MSP
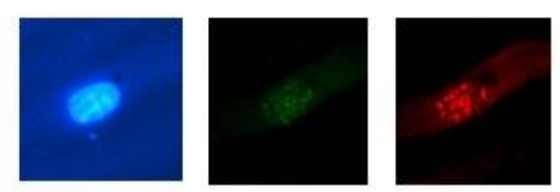

$\mathrm{BC}_{3}$ OSP1
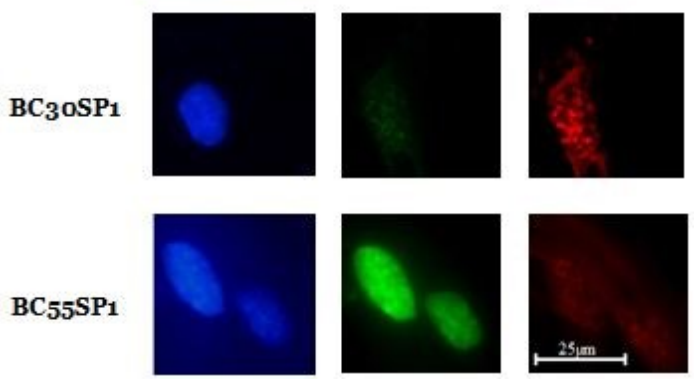

Figure 1. Cell lines, stained with DAPI, with antibodies to histones $53 \mathrm{BP} 1$ and $\gamma \mathrm{H} 2 \mathrm{AX} 30$ minutes after irradiation 
The term "foci" is used for DNA repair sites that occur after DNA damage, when proteins participating in the response or their modified forms are synthesized, which accumulate at the sites of doublestranded DNA breaks and can be visualized using immunocytochemistry.

To assess the reparative capabilities of DNA, cell lines were exposed to ionizing radiation at a dose of 2 Gy on a RAP-150 / 300-14 setting (Russia). Then cells were fixed after 30 minutes, 1.5 hours, 6 hours and 24 hours. Intact cells were used as a control (zero point) for each line. Time ranges and doses were selected based on published data [11] and the experience of the Laboratory of Radiation Cytology, Institute of Cytology RAS, St. Petersburg.

\subsection{Immunocytochemical analysis}

Using the indirect immunofluorescence method, the cell lines were stained with antibodies to histones $53 \mathrm{BP} 1$ and $\gamma \mathrm{H} 2 \mathrm{AX}$.

According to the standard protocol and usage annotations of antibodies, the cells were fixed on ice with a $4 \%$ formaldehyde solution for 10 minutes at $+40 \mathrm{C}$ followed by the fixation with ice-cold $70 \%$ ethanol solution in PBS (15 min at room temperature), washed with a solution of PBS, permeabilized with a $1.5 \% \mathrm{X}-100$ triton solution in PBS (15 $\mathrm{min}$ at room temperature), washed with a PBS solution 3 times for 5 minutes and incubated in a blocking solution of $5 \%$ BSA in PBS ( $1 \mathrm{~h}$ at room temperature), followed by washing with PBS. As the first antibodies, mouse IgG to $\gamma \mathrm{H}$ 2AX (ThermoFisher, USA), and rabbit IgG to the 53BP1 protein (Abcam, UK), Goat antibodies against mouse or rabbit IgG conjugated with Alexa Fluor 488 and 546 (Invitrogen, USA) at a 1:500 dilution were used as the second antibodies. To prevent the rapid burnout of immunofluorescence dyes, the preparations were enclosed in antifading using SlowFade Gold Antifade reagent with DAPI (Invitrogen, USA).

\subsection{Microscopy}

Images from stained preparations were obtained using an AXIOVERT 200M fluorescent inverted microscope (Carl Zeiss AG, Germany) equipped with a Leica DFC 420 camera and a×40/0.75 lens. In each line, using the Fiji program, fluorescence intensity in 100 cells was determined.

\subsection{Statistical analysis}

For the parameter of statistical analysis, the standard deviation of the mean values was chosen. Statistical analysis was performed in Google Sheets and Excel.

\subsection{Foci parameters analysis}

To assess the reparative capabilities of DNA, the following foci parameters were studied: amount, area, and fluorescence intensity. The parameters were analyzed in the DARFI program, since it had the smallest subjective component among programs such as IPLab, Fiji, FoCo, and CellProfiller [11].

\section{RESULTS}

The reparative ability of cells can be assessed by such parameters as size, area and fluorescence intensity of foci. In cells of healthy donors, the maximum values of the number of foci are usually observed 30 minutes or 1.5 hours after exposure to ionizing radiation.

\subsection{Number of foci $53 B P 1$ and $\gamma H 2 A X$}

The highest average value of the number of foci $53 \mathrm{BP} 1$ is observed in cells of a healthy donor. The maximum values are detected at 30 minutes. 24 hours later, the average value of the number of foci $53 \mathrm{BP} 1$ was less than at the starting point.

In all other cell lines, the maximum of the average value of $53 \mathrm{BP} 1$ foci number are observed 1.5 hours after irradiation and are indistinguishable from a healthy donor's values 24 hours later.

It is interesting that the "zero point", that is, the average value of $53 \mathrm{BP} 1$ number foci in intact cells, is the same in all studied lines, except for the healthy donor line (N9SP). The zero point of the healthy donor line is significantly higher than in the other lines.

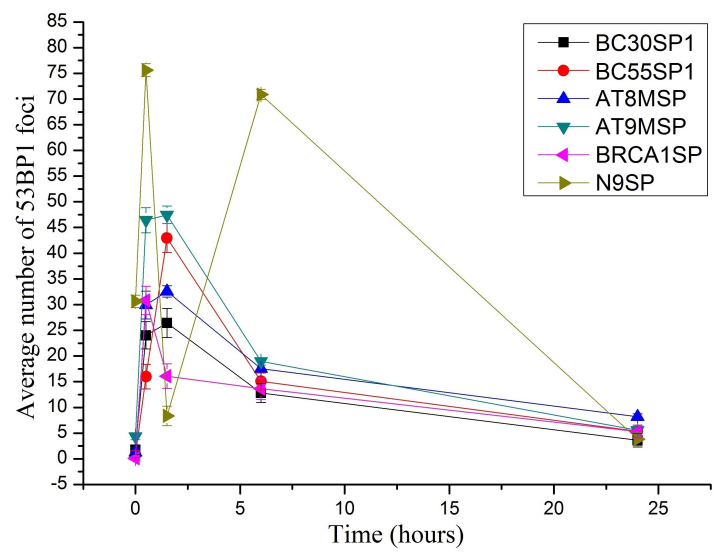

Figure 2. Average number of $53 \mathrm{BP} 1$ foci

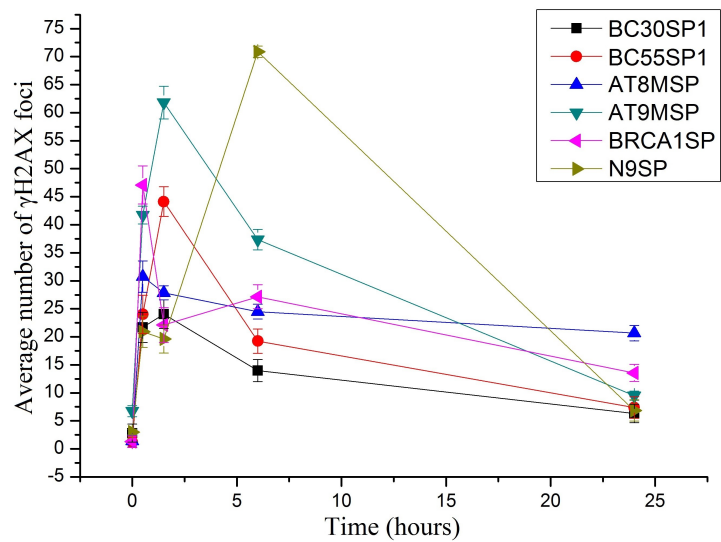

Figure 3. Average number of $\gamma \mathrm{H} 2 \mathrm{AX}$ foci

The repair curves of the $53 \mathrm{BP} 1$ foci number in patients with breast cancer are different and suggest about of more serious DNA damage in the young BC patient. The curve of the younger patient with breast cancer changed less sharply since the cells did not react so quickly to DNA damage although, at the same time, they did not form a "plateau" at high values of foci. 
Also the repair curves of $\mathrm{BC}$ patients and persons with medical history of cancer were different. The increase in mean values of $53 \mathrm{BP} 1$ foci in the lines of persons with medical history of cancer from zero point to the point 30 minutes after irradiation was greater than in cells of patients with breast cancer, but lower than in cells of a healthy donor.

The cells of a healthy donor also have the highest average number of $\gamma \mathrm{H} 2 \mathrm{AX}$ foci. In contrast to the average values of the foci $53 \mathrm{BP} 1$, the maximum number of foci is observed in the N9SP line 6 hours after irradiation. The zero point in terms of the number of $\gamma \mathrm{H} 2 \mathrm{AX}$ foci in all studied cell lines, except for the AT9MSP line, is also the same. The mean value of $\gamma \mathrm{H} 2 \mathrm{AX}$ foci at the zero point in the AT9MSP line is significantly higher (2 times) than in the other lines. A day after irradiation, the mean value of $\gamma \mathrm{H} 2 \mathrm{AX}$ foci in the AT9MSP line did not differ from the values in the line of a healthy donor and patients with breast cancer. The average values of the number of $\gamma \mathrm{H} 2 \mathrm{AX}$ foci one day after irradiation in the AT8MSP and BRCA1SP lines are significantly higher than in the other lines. The repair curve of the AT8MSP line from the time point 1.5 hours after irradiation reached a plateau and did not return to the initial values. This fact demonstrates how different the repair capacity of heterogeneous carriers of the ATM gene mutation can be.

\subsection{Fluorescence intensity of $53 \mathrm{BP} 1$ and $\gamma \mathrm{H} 2 \mathrm{AX}$ foci}

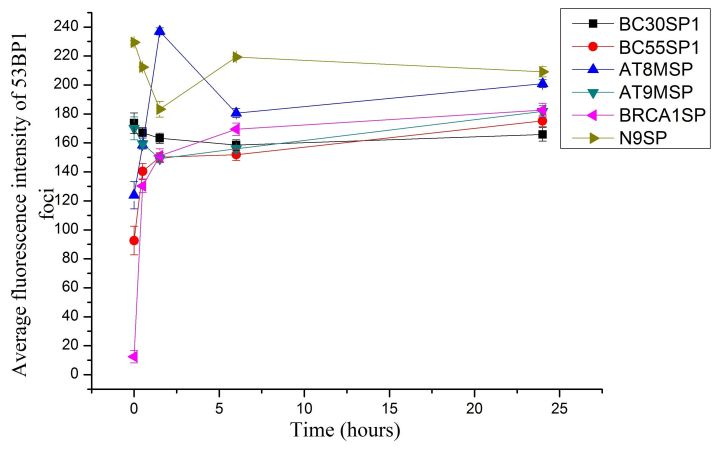

Figure 4. Average fluorescence intensity of $53 \mathrm{BP} 1$ foci

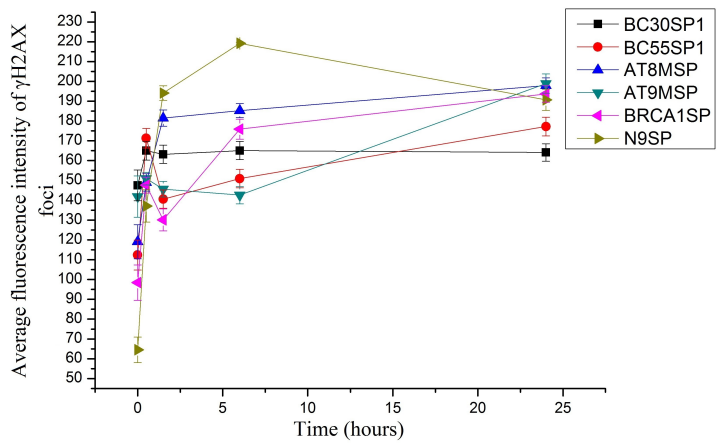

Figure 5. Average fluorescence intensity of $\gamma \mathrm{H} 2 \mathrm{AX}$ foci

The average fluorescence intensity of $53 \mathrm{BP} 1$ foci all cell lines are differ at different time intervals. The highest mean value of the fluorescence intensity of $53 \mathrm{BP} 1$ foci at zero point is demonstrate by cells of a healthy donor. Also, the healthy donor's line show the highest values of fluorescence intensity at all time intervals, except for the time point 1.5 hours after irradiation, when values are lower than in the AT8MSP cell line. During the first hour and a half after irradiation, a sharper decrease in the mean fluorescence intensity of $53 \mathrm{BP} 1$ foci observe, then an increase in the repair curve to values equal to 30 minutes after irradiation.

The mean fluorescence intensity at this point in the AT8MSP line is slightly but significantly higher than in the healthy donor line. The smallest range of differences in the mean fluorescence intensity of $53 \mathrm{BP} 1$ foci observe in AT9MSP and BC30SP1 cell lines.

The highest average fluorescence intensity of $\gamma \mathrm{H} 2 \mathrm{AX}$ foci was demonstrated by cells of a healthy donor 1.5 and 6 hours after irradiation. In contrast to the mean values of $53 \mathrm{BP} 1$ foci, the repair curve had the broadest range of mean values of the $\gamma \mathrm{H} 2 \mathrm{AX}$ fluorescence intensity. Cells of N9SP line had the lowest value at the zero point of all lines and, one 24 hours after irradiation the average of $\gamma \mathrm{H} 2 \mathrm{AX}$ foci number did not return to the initial values. Interestingly, the zero points of the rest of the cell lines are approximately the same in terms of fluorescence intensities of $53 \mathrm{BP} 1$ and $\gamma \mathrm{H} 2 \mathrm{AX}$ foci. The next highest fluorescence intensity values for foci $53 \mathrm{BP} 1$ and $\gamma \mathrm{H} 2 \mathrm{AX}$ are the lines of a 30 years old breast cancer patient and AT9MSP.

\subsection{Average area of $53 B P 1$ and $\gamma H_{2} A X$ foci}

Foci areas were measured in pixels. The cells of a healthy donor and a patient with the 5382incC mutation in the BRCA1 gene showed the smallest mean value of the $53 \mathrm{BP} 1$ foci area. This fact is consistent with the fact that in intact cells of a healthy donor, DNA damage arising in the cell's life cycle is not critical and the repair system copes with it. A day after irradiation, the average area of $53 \mathrm{BP} 1$ foci in these lines was the highest. This is probably due to the fact that a day after DNA damage, mainly large foci remain in a healthy cell as the cell successfully fixes the small ones. In other cell lines, the average area of $53 \mathrm{BP} 1$ foci at zero point was significantly higher and decreased one day after irradiation.

The intact cells of breast cancer patients had the largest foci of $53 \mathrm{BP} 1$, which confirms the fact of serious disruptions in the processes of DNA repair. Moreover, in intact cells of a 55-year-old patient with breast cancer, the area of foci was 1.5 times significantly higher than in intact cells of a 30-year-old patient with breast cancer.

After irradiation, the average area of $53 \mathrm{BP} 1$ foci in all cell lines showed a tendency towards a decrease in for the first 6 hours, most likely due to the emergence of many new foci. Then, a cell either repaired these foci, or these foci grew into larger ones.

An overall picture for the mean values of the $\gamma \mathrm{H} 2 \mathrm{AX}$ foci area in the cells of a healthy donor was very similar to the mean values of the $53 \mathrm{BP} 1$ foci area, except for the time point of 30 minutes after irradiation. The data of $53 \mathrm{BP} 1$ foci area formed a peak downward, while the data of the average values of the $\gamma \mathrm{H} 2 \mathrm{AX}$ foci area formed a sharp upward peak at this point. The data of values of the $\gamma \mathrm{H} 2 \mathrm{AX}$ and $53 \mathrm{BP} 1$ foci area 30 minutes after irradiation in the cell lines of 
breast cancer patients formed downward peak. Moreover, in the cells of the older patient with breast cancer, a sharper decrease in the area of foci (two times) was observed in comparison to the line of the younger patient with breast cancer. The average values of $\gamma \mathrm{H} 2 \mathrm{AX}$ foci area of the younger patient with breast cancer continued to decrease smoothly 1.5 hours after irradiation and by 6 hours after irradiation it began to rise smoothly. This cell line had the smallest range in terms of the $\gamma \mathrm{H} 2 \mathrm{AX}$ foci area and was at rather high values. This fact allows us to conclude the presence of more serious disruptions of DNA repair in the cells of the younger patient with breast cancer than in the cells of the older patient. Interestingly, in the cells of persons with a medical history of cancer development in intact cells, the area of $\mathrm{\gamma H} 2 \mathrm{AX}$ foci was significantly higher than in cells of a healthy donor. The largest excess of the $\gamma \mathrm{H} 2 \mathrm{AX}$ foci area values was greater in the BRCA1SP line. In all cell lines with medical history of cancer 24 hours after irradiation the $\gamma \mathrm{H} 2 \mathrm{AX}$ foci areas tended to decrease and had the lowest values. This fact may indicate that the cell's DNA repair systems are disrupted and cannot cope with minor DNA damage.

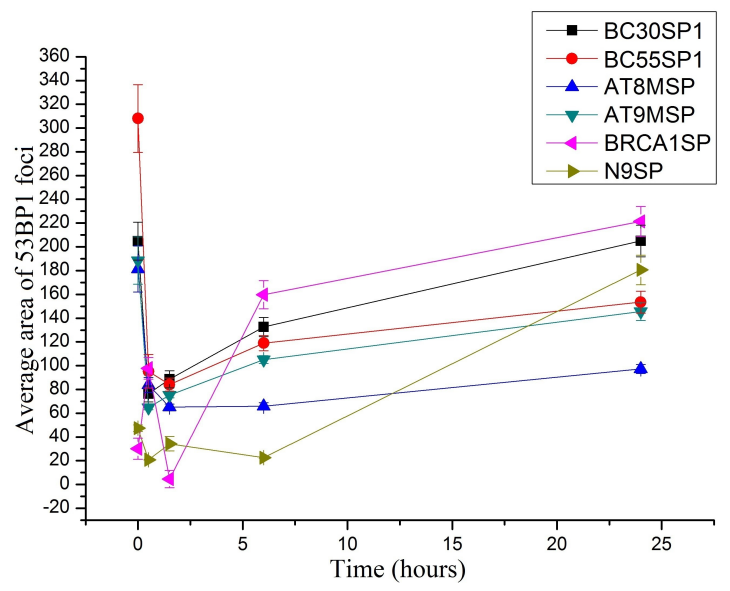

Figure 6. Average area of $53 \mathrm{BP} 1$ foci

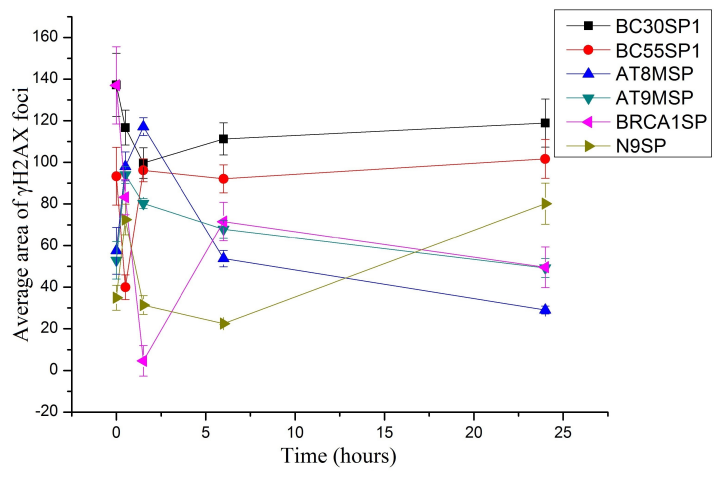

Figure 7. Average area of $\gamma \mathrm{H} 2 \mathrm{AX}$ foci

\section{CONCLUSION}

For a more complete understanding of DNA repair processes in the cell, it is necessary to compare the results in terms of the amount, intensity of fluorescence, and the area of foci. In terms of the number of foci $53 \mathrm{BP} 1$ and $\gamma \mathrm{H} 2 \mathrm{AX}$ the values had the widest range of values. The width of the range of data average number, intensity and area of fluorescence $53 \mathrm{BP} 1$ and $\gamma \mathrm{H} 2 \mathrm{AX}$ foci of patients with breast cancer at different time intervals differ from each other. The younger patient with breast cancer had a narrower range of focus parameters. This fact once again confirms the presence of more serious DNA repair process disruptions in the younger patient. Also, the numbers and fluorescence intensity of $53 \mathrm{BP} 1$ foci of persons with medical history of cancer and healthy donor cell lines differ. However, between patients with breast cancer those values do not differ. Interestingly, histone $\gamma \mathrm{H} 2 \mathrm{AX}$ data in all cell lines differ. The results obtained allow us to conclude that histone $\gamma \mathrm{H} 2 \mathrm{AX}$ is a more specific marker of breast cancer than $53 \mathrm{BP} 1$ protein.

Acknowledgements: The work was supported by Grants Councils of the President of the Russian Federation $M K-3638.2019 .7$. The authors would like to thank for the assistance in working to Daria Pasekova and Aleksey Manikhas.

\section{REFERENCES}

1. M. Majidinia, B. Yousefi, "DNA repair and damage pathways in breast cancer development and therapy," DNA Repair, vol. 54, pp. 22 - 29, Jun. 2017.

DOI: 10.1016/j.dnarep.2017.03.009 PMid: 28437752

2. J. H. Hoeijmakers, "DNA damage, aging, and cancer," N. Engl. J. Med., vol. 361, no. 15, pp. 1475 - 1485, Oct. 2009.

DOI: 10.1056/NEJMrao804615 PMid: 19812404

3. G. Ciriello et al., "Emerging landscape of oncogenic signatures across human cancers," Nat. Genet., vol. 45, no. 10, pp. 1127 - 1133, Oct. 2013.

DOI: $10.1038 / \mathrm{ng} .2762$

PMid: 24071851 PMCid: PMC4320046

4. H. C. Reinhardt, H. Jiang, M. T. Hemann, M. B. Yaffe, "Exploiting synthetic lethal interactions for targeted cancer therapy," Cell Cycle, vol. 8, no. 19, pp. $3112-3119$, Oct. 2009.

DOI: $10.4161 /$ cc.8.19.9626

PMid: 19755856 PMCid: PMC 3057180

5. O. Moran et al., "Revisiting breast cancer patients who previously tested negative for BRCA mutations using a 12-gene panel," Breast Cancer Res. Treat., vol. 161, no. 1, pp. 135 - 142, Jan. 2017.

DOI: $10.1007 /$ s10549-016-4038-y PMid: 27798748

6. S. Sriramulu et al., "A review on role of ATM gene in hereditary transfer of colorectal cancer," Acta Biomed., vol. 89, no. 4, pp. $463-469$, Jan. 2019.

DOI: $10.23750 /$ abm.v89i4.6095

PMid: 30657113 PMCid: PMC6502098

7. A. Tavera-Tapia et al., "Almost $2 \%$ of Spanish breast cancer families are associated to germline pathogenic mutations in the ATM gene," Breast Cancer Res. Treat., vol. 161, no. 3, pp. 597 - 604, Feb. 2017. DOI: $10.1007 / \mathrm{s} 10549-016-4058-7$ PMid: 27913932

8. S. M. Noordermeer et al., "The shieldin complex mediates 53BP1-dependent DNA repair," Nature, vol. 560, no. 7716, pp. 117 - 121, Aug. 2018.

DOI: $10.1038 / \mathrm{s} 41586-018-0340-7$

PMid: 30022168 
A. Nozdracheva et al., Markers of the cell's response to DNA damage, RAP Conf. Proc., vol. 5, 2020, 38-43

PMCid: PMC6141009

9. G. D. Gregoriis et al., "DNA repair genes PAXIP1 and TP53BP1 expression is associated with breast cancer prognosis," Cancer Biol. Ther., vol. 18, no. 6, pp. $439-449$, Jun. 2017.

DOI: $10.1080 / 15384047.2017 .1323590$

PMid: 28475402

PMCid: PMC5536937

10. Y. Li, G. Zhang, "Network-based characterization and prediction of human DNA repair genes and pathways," Scientific Reports, vol. 7, pp. 1 - 8, Apr. 2017.

DOI: $10.1038 /$ srep45714

PMid: 28368026

PMCid: PMC5377940
11. A. Nozdracheva, R. Ushakov, N. Pleskach, M. Kuranova, "Analysis of the capabilities of the programs Fiji, iPLab and DARFI in the study of DNA repair abilities in the cells of patients with a mosaic form of ataxia telangiectasia," in Proc. Int. Conf. Radiation Applications (RAP 2019), Belgrade, Serbia, 2019, pp. $1-6$.

DOI: $10.37392 /$ RapProc.2019.01

12. M. Kuranova et al., R. "Features of DNA repair in dermal fibroblasts of patients with ataxia-telangiectasia with mosaic manifestation of the active form of ATM kinase," Genes \& Cells, tome XIV, №1, 2020, pp. $50-59$.

DOI: $10.23868 / 202003007$ 